\title{
AN INTERNAL-STATE-VARIABLE BASED VISCOELASTIC-PLASTIC MODEL FOR POLYMERS
}

\author{
CYPRIAN SUCHOCKI \\ Warsaw University of Technology, Department of Mechanics and Armament Technology, Warsaw, Poland \\ e-mail: c.suchocki@imik.wip.pw.edu.pl
}

In this study, a new viscoelastic-plastic constitutive model which has been formulated by utilizing the formalism of stress-like internal state variables is introduced. The developed constitutive equation allows for a good description of the inelastic material response of polymeric materials over a wide range of strain rates. An algorithm for numerical integration of the model equations has been derived. The FE implementation of the constitutive equation is widely discussed and the results of solving several exemplary problems are presented.

Keywords: polymers, rheology, constitutive equation, finite element method

\section{Nomenclature}

$b \quad-$ Knowles material parameter

$\mathbf{B}, \overline{\mathbf{B}} \quad-$ left Cauchy-Green (C-G) and isochoric left C-G deformation tensor

$\mathbf{C}, \overline{\mathbf{C}} \quad-$ right and isochoric right $\mathrm{C}-\mathrm{G}$ deformation tensor

$\mathcal{C}, \mathcal{C}^{v e-p}-$ elasticity tensor and viscoelastic-plastic material tensor

$\mathcal{C}^{\tau c}-$ material tensor related to convected stress rate

$\mathcal{C}^{M J} \quad-$ material Jacobian tensor used by Abaqus

D $\quad-$ strain rate tensor

$D_{1} \quad-\quad$ inverse of bulk modulus

$\mathbf{e}_{k} \quad-\quad$ unit vector of Cartesian base, $k=1,2,3$,

$\mathbf{F}, \overline{\mathbf{F}} \quad-$ deformation gradient and isochoric deformation gradient tensor

$\mathbf{H}_{k} \quad-\quad$ viscoelastic overstress tensor $k=1,2, \ldots, N$

$\widetilde{\mathbf{H}}_{k} \quad-$ endochronic overstress tensor $k=1,2, \ldots, P$

I $\quad-$ fourth order identity tensor

$\mathbf{I}_{\mathbf{C}^{-1}} \quad-$ fourth order identity tensor in reference configuration

$\mathcal{I} \quad-$ auxiliary fourth order tensor

$I_{k}, \bar{I}_{k} \quad-$ algebraic invariants of right and of isochoric right $\mathrm{C}-\mathrm{G}$ deformation tensor, $k=1,2,3$

$\bar{J}_{k} \quad-\quad$ algebraic invariants of material time derivative of isochoric right C-G deformation tensor, $k=1,2,3$

$J \quad-$ Jacobian determinant

L $\quad-$ velocity gradient tensor

Q $\quad-$ orthogonal tensor

$\mathbf{S}, \overline{\mathbf{S}} \quad-$ second Piola-Kirchhoff $(\mathrm{P}-\mathrm{K})$ and auxiliary second P-K total stress tensor

$\mathbf{S}_{0} \quad-$ second P-K elastic stress tensor

$\mathbf{T} \quad-$ Lagrange total stress tensor

$t \quad-\quad$ time since loading

$U \quad-\quad$ volumetric stored elastic energy potential 


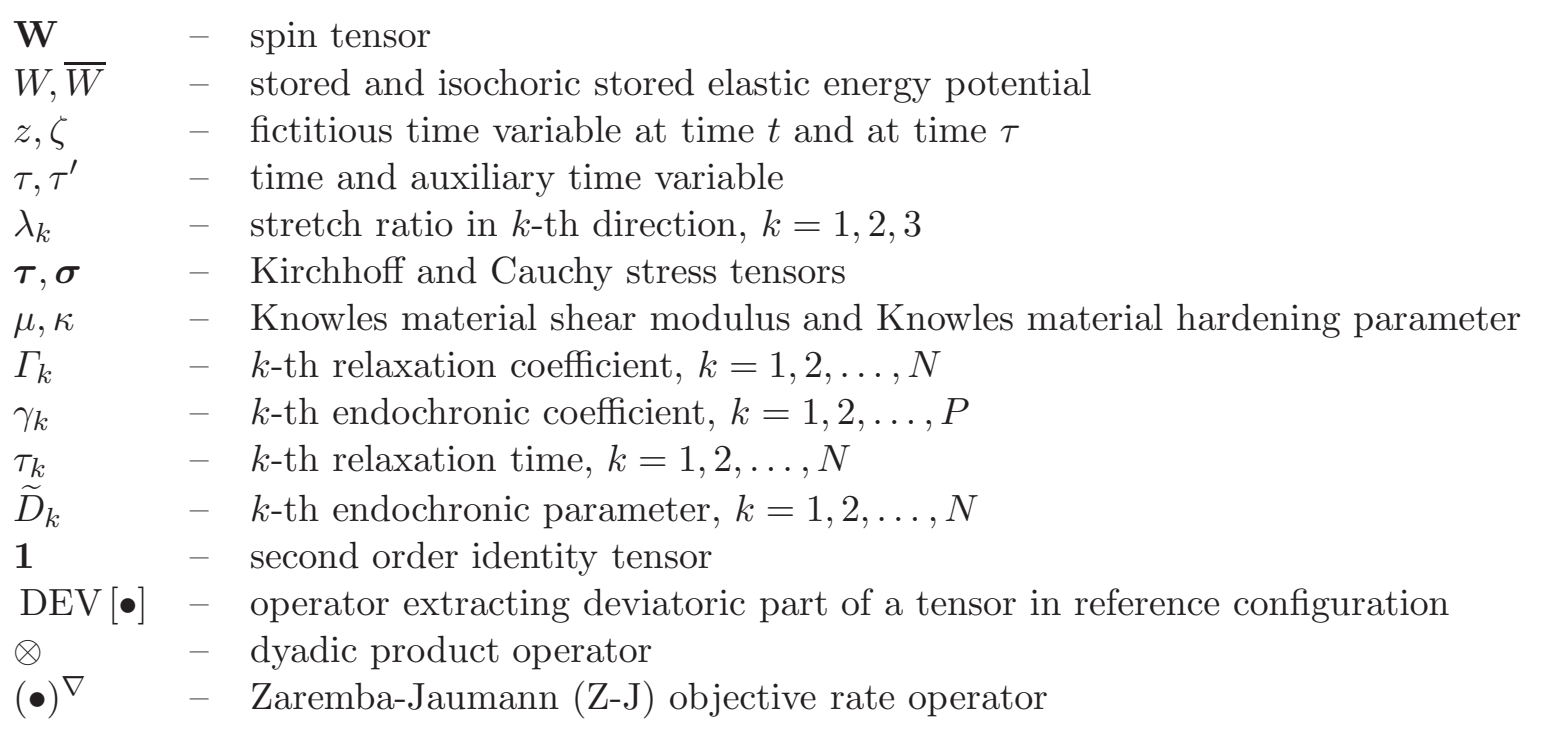

\section{Introduction}

In recent years, various constitutive equations of viscoelasticity and viscoplasticity have been developed in order to describe the mechanical properties of thermoplastic polymers and resins. Ayoub et al. (2011) presented a viscoplastic model aimed at capturing the large strain response of polyethylene over a wide range of strain rates. A better approximation of the stress-strain relation of polyethylene, however for a narrower range of the strain rates, was achieved by Bergström and Bischoff (2010) who developed the so-called three network viscoplastic model. Abdul Hameed et al. (2014) formulated a constitutive model based on the multiplicative split of the deformation gradient. It was further used to simulate the monotonic stress-strain curves of polyethylene. Bardella (2001) developed a nonlinear viscoelastic constitutive model oriented for the description of the epoxy resin behavior during cyclic loadings and creep.

Instead of modeling the mechanical behavior of a polymer over the entire range of physically possible strains, some researchers focus on improving the constitutive equations for the spectrum of small and moderate deformations which are usually experienced by the structural elements. Drozdov and Christiansen (2007) developed a viscoplastic constitutive model aimed at predicting the stresses in high density polyethylene (HDPE) subjected to the cyclic loadings in tension. Hassan et al. (2011) utilized an overstress-based viscoplasticity theory to model the hysteresis loops of ultra high molecular weigth polyethylene (UHMWPE) for the maximum strains equal to 10\%. Ben Hadj Hamouda et al. (2007) developed a viscoplastic model to capture the nonlinear stress-strain relation, creep and relaxation of the medium density polyethylene (MDPE). Monotonic stress-strain curves with the maximum strain of $12 \%$ were analyzed. Krairi and Doghri (2014) proposed a model utilizing the additive split of the strain tensor into viscoelastic and viscoplastic components. Monotonic and cyclic stress-strain curves were approximated for both polyamide (PA) and HDPE with the maximum axial strain of $14 \%$.

Constitutive theories based on the endochronic plasticity theory enjoy recently an increasingly high interest. This theory was originally developed by Pipkin and Rivlin (1965). Kästner et al. (2012) proposed a viscoplastic constitutive equation for polyprophylene (PP) based on the generalized Maxwell mechanistic model. The polymer viscosity was assumed to depend on the non-equilibrium overstress while endochronic plasticity was used to help modeling the hysteresis loop. Strains up to $5 \%$ in tension were analyzed and infinitesimal strain tensor was used. This approach was later generalized to large displacements and large rotations by Alkas Yonan et al. (2013) for the purpose of modeling the inelastic response of polyvinylchloride (PVC). 
All the mentioned above constitutive theories require determination of about twenty material parameters. The numerical algorithms used for integrating the models are usually complicated.

In the current study, a new viscoelastic-plastic constitutive model for thermoplastics and resins is presented. The model is an extension of the popular nonlinear viscoelasticity theory proposed by Holzapfel (2010) and widely used in FE codes. The formalism of internal stress-like state variables previously used by Suchocki (2013) to develop a nonlinear viscoelastic model for polymers is extended by taking advantage of the endochronic plasticity framework (Pipkin and Rivlin, 1965). The common assumptions of isothermal deformations and treating the effects of volumetric viscoelasticity as negligible have been applied. It was experimentally observed that irreversible volume changes are usually small (e.g. Wu, 2005). Thus, the postulate of plastic incompressibility is well established and utilized by the classical theory of plasticity. This assumption is adopted in the constitutive model formulated in the present study for sake of simplicity. The constitutive equation has a modular structure which enables adjusting it for a specific polymeric material. It is applied to capture the inelastic behavior of polyethylene. For that purpose the specific values of the material parameters have been determined. It is found that the proposed constitutive model accurately reproduces the stress relaxation curve and the hysteresis loop for the deformation rate magnitude from the interval $0.0005-0.05 \mathrm{~s}^{-1}$. The constitutive equation is discretized and an algorithm for efficient numerical computations is presented. The implementation of the model into the FE software Abaqus is discussed.

\section{Decoupled finite elasticity}

In this section, the basic notions of hyperelasticity are presented. A material is called hyperelastic if it posseses a stored-energy function $W=W(\mathbf{C})$ (Holzapfel, 2010). In the case of material isotropy, this function must be invariant with respect to a rotation $\mathbf{Q}$, i.e.

$$
W(\mathbf{C})=W\left(\mathbf{Q} \mathbf{C} \mathbf{Q}^{\mathrm{T}}\right)
$$

This requirement is fulfilled if $W$ is a function of algebraic invariants of $\mathbf{C}$.

In terms of FEM, it is profitable to decouple the volumetric and isochoric responses within the constitutive model. This is facilitated by utilizing proper multiplicative decomposition of the deformation gradient tensor $\mathbf{F}$

$$
\mathbf{F}=\mathbf{F}_{v o l} \overline{\mathbf{F}} \quad \mathbf{F}_{\text {vol }}=J^{\frac{1}{3}} \mathbf{1} \quad \overline{\mathbf{F}}=J^{-\frac{1}{3}} \mathbf{F} \quad \overline{\mathbf{C}}=\overline{\mathbf{F}}^{\mathrm{T}} \overline{\mathbf{F}}=J^{-\frac{2}{3}} \mathbf{C}
$$

where $\mathbf{F}_{\text {vol }}$ and $\overline{\mathbf{F}}$ represent purely volumetric and isochoric deformations, respectively. Consequently, the algebraic invariants of $\overline{\mathbf{C}}$

$$
\bar{I}_{1}=\operatorname{tr} \overline{\mathbf{C}} \quad \bar{I}_{2}=\frac{1}{2}\left[(\operatorname{tr} \overline{\mathbf{C}})^{2}-\operatorname{tr} \overline{\mathbf{C}}^{2}\right] \quad \bar{I}_{3}=\operatorname{det} \overline{\mathbf{C}}=1
$$

The stored-energy function is formulated in a decoupled form

$$
W(\mathbf{C})=U(J)+\bar{W}(\overline{\mathbf{C}})
$$

where $U$ and $\bar{W}$ are the stored-energy components associated with the volumetric and isochoric deformations, respectively. The stored-energy function as given by (2.4) results in

$$
\mathbf{S}=J p \mathbf{C}^{-1}+J^{-\frac{2}{3}} \operatorname{DEV}[\overline{\mathbf{S}}]
$$

where

$$
p=\frac{\partial U}{\partial J} \quad \operatorname{DEV}[\overline{\mathbf{S}}]=\overline{\mathbf{S}}-\frac{1}{3}(\overline{\mathbf{S}} \cdot \overline{\mathbf{C}}) \overline{\mathbf{C}}^{-1} \quad \overline{\mathbf{S}}=2 \frac{\partial \bar{W}}{\partial \overline{\mathbf{C}}}
$$

and $\operatorname{DEV}[\bullet]=[\bullet]-\frac{1}{3}([\bullet] \cdot \overline{\mathbf{C}}) \overline{\mathbf{C}}^{-1}$ is an operator extracting a deviator of a tensor in the reference configuration, whereas $\overline{\mathbf{S}}$ is an auxiliary stress tensor. 


\section{Internal-state-variable based viscoelastic-plastic model}

The total stress is assumed in the form of a sum

$$
\mathbf{S}=\mathbf{S}_{0}+\sum_{k=1}^{P} \widetilde{\mathbf{H}}_{k}+\sum_{j=1}^{N} \mathbf{H}_{j}
$$

where the components $\mathbf{S}_{0}$ and $\widetilde{\mathbf{H}}_{k}(k=1,2, \ldots, P)$ describe the equilibrium material response, whereas $\mathbf{H}_{j}(j=1,2, \ldots, N)$ account for the viscoelastic effects. $\mathbf{S}_{0}$ is taken in the decoupled form, i.e.

$$
\mathbf{S}_{0}=\mathbf{S}_{0}^{v o l}+\mathbf{S}_{0}^{i s o} \quad \mathbf{S}_{0}^{v o l}=J \frac{\partial U}{\partial J} \mathbf{C}^{-1} \quad \mathbf{S}_{0}^{i s o}=2 J^{-\frac{2}{3}} \operatorname{DEV}\left[\frac{\partial \bar{W}}{\partial \overline{\mathbf{C}}}\right]
$$

The evolution of overstresses $\widetilde{\mathbf{H}}_{k}$ is governed by the equations of the form

$$
\dot{\widetilde{\mathbf{H}}}_{k}+\frac{1}{\widetilde{D}_{k} M(|\dot{\mathbf{C}}|)} \widetilde{\mathbf{H}}_{k}=\gamma_{k} \dot{\mathbf{S}}_{0}^{i s o} \quad k=1,2, \ldots, P
$$

where

$$
M(|\dot{\mathbf{C}}|)=\bar{J}_{2}^{-\frac{1}{2}} \quad \bar{J}_{2}=\operatorname{tr} \dot{\mathbf{C}}^{2}
$$

This specific choice of $M$ enables one to eliminate the time from Eq. (3.3), thus making the evolution of $\widetilde{\mathbf{H}}_{k}$ rate-independent. By integrating Eq. (3.3), it is found that

$$
\widetilde{\mathbf{H}}_{k}(t)=\int_{0}^{z(t)} \gamma_{k} \mathrm{e}^{-\frac{z-\zeta}{\widetilde{D}_{k}}} \frac{\partial \mathbf{S}_{0}^{i s o}(\zeta)}{\partial \zeta} d \zeta
$$

where the following equalities are used

$$
\dot{\zeta}=\frac{1}{M(|\dot{\mathbf{C}}|)} \quad \zeta(\tau)=\int_{-\infty}^{\tau} \frac{d \tau^{\prime}}{M\left(\tau^{\prime}\right)} \quad \zeta(t)=z
$$

Substituting Eqs (3.4) into Eq. (3.6) 2 leads to the result

$$
\zeta(\tau)=\int_{-\infty}^{\tau}\left[\dot{\overline{\mathbf{C}}}\left(\tau^{\prime}\right) \cdot \dot{\overline{\mathbf{C}}}\left(\tau^{\prime}\right)\right]^{\frac{1}{2}} d \tau^{\prime} \quad(\dot{\bullet})=\frac{d}{d \tau^{\prime}}(\bullet)
$$

which in turn gives

$$
\zeta(\tau)=\int_{-\infty}^{\tau}\left[d \overline{\mathbf{C}}\left(\tau^{\prime}\right) \cdot d \overline{\mathbf{C}}\left(\tau^{\prime}\right)\right]^{\frac{1}{2}}
$$

The variable $\zeta$ is a fictious internal time associated with the material. For this reason the overstresses defined by Eq. (3.5) are called endochronic. Equation (3.5) is a special case of the stress-strain relation proposed by Pipkin and Rivlin (1965). The stress tensor $\mathbf{S}_{0}$ together with the endochronic overstresses $\widetilde{\mathbf{H}}_{k}(k=1,2, \ldots, P)$ form up the elasto-plastic part of the constitutive equation which is responsible for reproducing the hysteresis loop of the modeled 
polymeric material. The strain rate effects are captured by the viscoelastic overstresses $\mathbf{H}_{j}$ $(j=1,2, \ldots, N)$ which evolve in time according to the following differential equation

$$
\dot{\mathbf{H}}_{j}+\frac{1}{\tau_{j}} \mathbf{H}_{j}=\Gamma_{j} \dot{\mathbf{S}}_{0}^{i s o} \quad j=1,2, \ldots, N
$$

After time integrating Eq. (3.9), one obtains

$$
\mathbf{H}_{j}(t)=\int_{-\infty}^{t} \Gamma_{j} \mathrm{e}^{-\frac{t-\tau}{\tau_{j}}} \frac{\partial \mathbf{S}_{0}^{i s o}(\tau)}{\partial \tau} d \tau
$$

For the purpose of modeling the mechanical properties of thermoplastics and resins, the stored-energy function $\bar{W}$ is adopted in the form proposed by Knowles (1977). The advantages of using the isochoric Knowles function were discussed by Suchocki (2011). The volumetric strain energy is assumed in the standard form, thus

$$
\bar{W}=\frac{\mu}{2 b}\left\{\left[1+\frac{b}{\kappa}\left(\bar{I}_{1}-3\right)\right]^{\kappa}-1\right\} \quad U=\frac{1}{D_{1}}(J-1)^{2}
$$

Summing up, the developed constitutive equation utilizes four elasticity constants, $2 P$ endochronic plasticity constants and $2 N$ constants of viscoelasticity all of which are independent.

\section{Discretization of constitutive equation}

Below a numerical algorithm for the incremental integration of the developed model is introduced. For the time increment $n+1$, the total stress is, according to Eq. (3.1), given by the equation

$$
\mathbf{S}_{n+1}=\mathbf{S}_{0 n+1}+\sum_{k=1}^{P} \widetilde{\mathbf{H}}_{k n+1}+\sum_{j=1}^{N} \mathbf{H}_{j n+1}
$$

The reccurence-update formula for the integration of Eq. (3.3) is obtained using the central difference method, thus the following approximations are utilized

$$
\left.\dot{y}\right|_{n+\frac{1}{2}} \approx \frac{y_{n+1}-y_{n}}{\Delta t}=\left.\frac{\Delta y_{n+1}}{\Delta t} \quad y\right|_{n+\frac{1}{2}} \approx y_{n}+\frac{1}{2} \Delta y_{n+1}
$$

Applying the rules given by Eqs (4.2) to Eq. (3.3) one obtains the following formula

$$
\widetilde{\mathbf{H}}_{k n+1}=\frac{\left(1-\frac{1}{\widetilde{D}_{k}} \frac{\Delta z_{n+1}}{2}\right) \widetilde{\mathbf{H}}_{k n}+\gamma_{k}\left(\mathbf{S}_{0 n+1}^{i s o}-\mathbf{S}_{0 n}^{i s o}\right)}{1+\frac{1}{\widetilde{D}_{k}} \frac{\Delta z_{n+1}}{2}}
$$

where

$$
\Delta z_{n+1}=\left(\Delta \overline{\mathbf{C}}_{n+1} \cdot \Delta \overline{\mathbf{C}}_{n+1}\right)^{\frac{1}{2}}
$$

The integration of Eq. (3.9) is performed by means of the reccurence-update formula developed by Taylor et al. (1970), i.e.

$$
\mathbf{H}_{j n+1}=\mathrm{e}^{-\frac{\Delta t}{\tau_{j}}} \mathbf{H}_{j n}+\Gamma_{j} \frac{1-\mathrm{e}^{-\frac{\Delta t}{\tau_{j}}}}{\frac{\Delta t}{\tau_{j}}}\left(\mathbf{S}_{0 n+1}^{i s o}-\mathbf{S}_{0 n}^{i s o}\right)
$$

Equations (4.1), (4.3) and (4.5) form up a discretized set of equations which can be utilized for numerical simulations. For the use of FEM, a material tangent stiffness tensor has to be defined in addition. The derivation of a tangent stiffness is discussed further in the text. 


\section{Exemplary application}

In order to check the ability of the developed viscoelastic-plastic constitutive equation to fit the experimental data, it has been employed to capture the mechanical properties of UHMWPE which is a popular biomaterial with numerous applications in orthopaedics, such as hip joint implants, elbow implants or artificial intervertebral discs for instance.

For the purpose of modeling the inelastic behavior of UHMWPE, a version of the developed constitutive equation has been adopted with $N=3$ viscoelastic overstresses and $P=1$ endochronic overstress. The graphic interpretation of the model can be seen in Fig. 1.

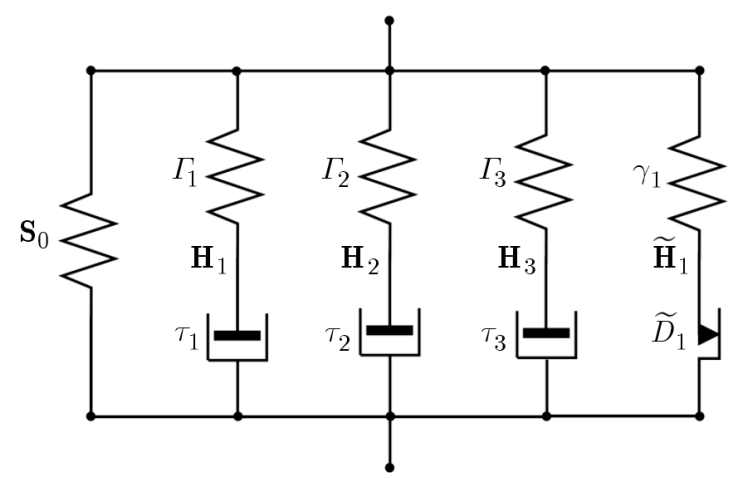

Fig. 1. Mechanical scheme of the rheological model assumed for UHMWPE

A number of mechanical tests have been performed on cylindrical specimens machined from a UHMWPE rod. The dimensions of the specimens are $\emptyset 17$ and $l_{0}=21 \mathrm{~mm}$. A medical grade polyethylene CHIRULEN 1050 has been used. All the experiments have been conducted on MTS Bionix 2500 testing machine at a constant room temperature of $20^{\circ} \mathrm{C}$.

A number of loading-unloading compression tests at a constant deformation rate $\dot{\lambda}$ has been performed. The used values of the deformation rate are $0.0005,0.005$ and $0.05 \mathrm{~s}^{-1}$ with $\lambda$ equal to 0.93 as the minimum stretch ratio. Furthermore, a relaxation test in compression has been carried out. The specimen has been loaded with a constant deformation rate of $0.003 \mathrm{~s}^{-1}$ and subsequently allowed to relax for 30 minutes.
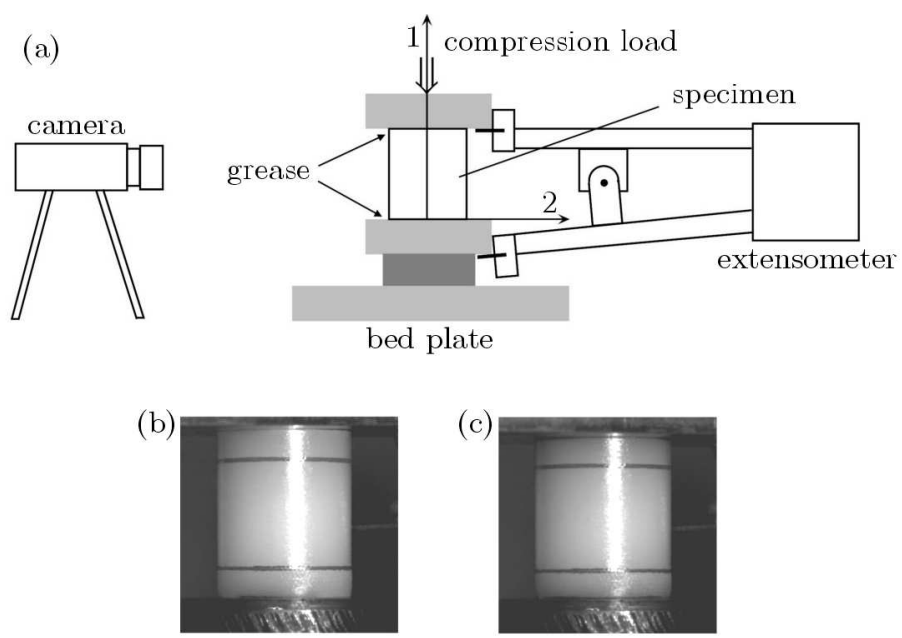

Fig. 2. (a) Experimental set-up. (b) View of undeformed specimen. (c) View of deformed specimen

The specimen stretch ratios in both axial an perpendicular directions have been measured using a video extensometer. The axial deformation has been at the same time measured with a strain gauge extensometer in order to verify the measured values. A sketch of the experimental setup can be seen in Fig. 2. A Poisson ratio of 0.46 has been determined, which justifies adopting an assumption of material incompressibility. 
The material parameters of the constitutive equation have been evaluated using the least squares method. Thus, the following error function has been minimized

$$
\mathcal{F}(\mathbf{p})=\sum_{k=1}^{P}\left[\left(T_{11}(\mathbf{p})\right)_{k}-\left(\widetilde{T}_{11}\right)_{k}\right]^{2}
$$

where $T_{11}(\mathbf{p})$ is the theoretical and $\widetilde{T}_{11}$ is the experimental Lagrange stress in the axial direction of the specimen at the time instant $t_{k}(k=1,2, \ldots, P)$. The column matrix $\mathbf{p}=\left[\mu, b, \kappa, \gamma_{1}, \widetilde{D}_{1}, \Gamma_{1}, \Gamma_{2}, \Gamma_{3}, \tau_{1}, \tau_{2}, \tau_{3}\right]^{\mathrm{T}}$ contains the material parameters being optimized. The experimental data from three loading-unloading tests at various deformation rates together with the data from the relaxation test have been utilized to form up the minimized objective function given by Eq. (5.1). The results of the curve fitting are shown in Fig. 3, whereas the determined values of the material constants of elasticity, viscoelasticity and plasticity have been collected in Table 1. The inverese of the bulk modulus $D_{1}$ does not participate in the optimization. In FE implementation, $D_{1}$ is set to a value corresponding to the bulk modulus high enough to account for the material near incompressibility.
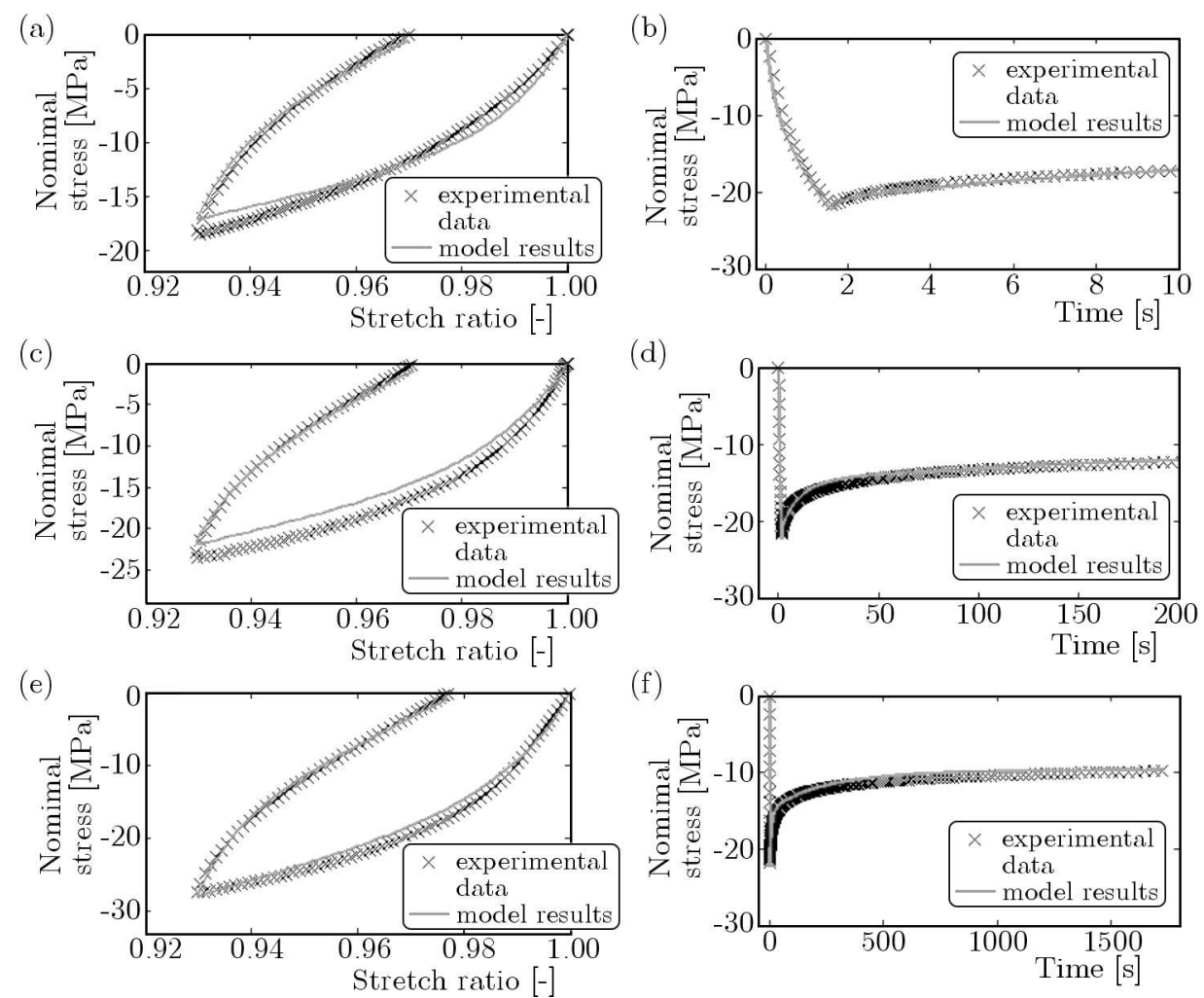

Fig. 3. Fitting of the viscoelastic-plastic constitutive model to the experimental data. Loading-unloading compression tests with the deformation rate of: (a) $0.0005 \mathrm{~s}^{-1}$, (b) $0.005 \mathrm{~s}^{-1}$, (c) $0.05 \mathrm{~s}^{-1}$ and stress relaxation tests with the relaxation time of: (d) $10 \mathrm{~s}$, (e) $200 \mathrm{~s}$, (f) $1700 \mathrm{~s}$

Table 1. Model parameters for UHMWPE

\begin{tabular}{|c|c|c|c|c|c|c|c|c|c|}
\hline \multicolumn{2}{|c|}{ Elastic constants } & \multicolumn{4}{|c|}{ Viscoelastic constants } & \multicolumn{4}{|c|}{ Plastic constants } \\
\hline$\mu$ & $52.56 \mathrm{MPa}$ & $\Gamma_{1}$ & 2.89 & $\tau_{1}$ & $0.16 \mathrm{~s}$ & $\gamma_{1}$ & 3.25 & $\widetilde{D_{1}}$ & 0.029 \\
\hline$b$ & 209.28 & $\overline{\Gamma_{2}}$ & 0.93 & $\tau_{2}$ & $8.803 \mathrm{~s}$ & & & & \\
\hline$\kappa$ & 0.81 & $\Gamma_{3}$ & 0.62 & $\overline{\tau_{3}}$ & $279.16 \mathrm{~s}$ & & & & \\
\hline$\overline{D_{1}}$ & $0.00033 \mathrm{MPa}^{-1}$ & & & & & & & & \\
\hline
\end{tabular}




\section{Finite element implementation}

The linearized constitutive equation used for the $\mathrm{FE}$ implementation is obtained by taking a directional derivative of Eq. (4.1) with respect to $\mathbf{C}_{n+1}$. Thus, for the $n+1$ increment

$$
\Delta \mathbf{S}_{n+1}=\mathcal{C}_{n+1}^{v e-p} \cdot \frac{1}{2} \Delta \mathbf{C}_{n+1} \quad \mathcal{C}_{n+1}^{v e-p}=2 \frac{\partial \mathbf{S}_{n+1}}{\partial \mathbf{C}_{n+1}}
$$

where the approximate material tangent tensor takes the form

$$
\mathcal{C}_{n+1}^{v e-p}=\mathcal{C}_{0 n+1}^{v o l}+\left[1+\sum_{k=1}^{P} \gamma_{k}\left(1+\frac{\Delta z_{n+1}}{2 \widetilde{D}_{k}}\right)^{-1}+\sum_{j=1}^{N} \Gamma_{j} \frac{1-\mathrm{e}^{-\frac{\Delta t}{\tau_{j}}}}{\frac{\Delta t}{\tau_{j}}}\right] \mathcal{C}_{0 n+1}^{i s o}
$$

The incremental constitutive rate equation given by Eq. (6.1) can be expressed using the Zaremba-Jaumann (Z-J) objective rate of the Kirchhoff stress $\boldsymbol{\tau}$, thus

$$
\boldsymbol{\tau}_{n+1}^{\nabla}=J_{n+1} \mathcal{C}_{n+1}^{M J} \cdot \Delta \mathbf{D}_{n+1}
$$

where the incremental Z-J objective rate of the Kirchhoff stress

$$
\boldsymbol{\tau}_{n+1}^{\nabla}=\Delta \boldsymbol{\tau}_{n+1}-\Delta \mathbf{W}_{n+1} \boldsymbol{\tau}_{n+1}-\boldsymbol{\tau}_{n+1} \Delta \mathbf{W}_{n+1}^{\mathrm{T}}
$$

and

$$
\begin{aligned}
& \Delta \mathbf{W}_{n+1}=\frac{1}{2}\left[\Delta \mathbf{F}_{n+1} \mathbf{F}_{n+1}^{-1}-\left(\Delta \mathbf{F}_{n+1} \mathbf{F}_{n+1}^{-1}\right)^{\mathrm{T}}\right] \\
& \Delta \mathbf{D}_{n+1}=\frac{1}{2}\left[\Delta \mathbf{F}_{n+1} \mathbf{F}_{n+1}^{-1}+\left(\Delta \mathbf{F}_{n+1} \mathbf{F}_{n+1}^{-1}\right)^{\mathrm{T}}\right] \\
& \Delta \mathbf{F}_{n+1}=\mathbf{F}_{n+1} \mathbf{F}_{n}^{-1}
\end{aligned}
$$

The material stiffness tensor takes the corresponding form

$$
\mathcal{C}_{n+1}^{M J}=\frac{1}{J_{n+1}}\left(\mathcal{C}_{n+1}^{\tau c}+\mathcal{I}_{n+1}\right)
$$

where

$$
\begin{aligned}
& \mathcal{C}_{n+1}^{\tau c}=\left(F_{i P} F_{j Q} F_{k R} F_{l S} \mathcal{C}_{P Q R S}^{v e-p}\right)_{n+1} \mathbf{e}_{i} \otimes \mathbf{e}_{j} \otimes \mathbf{e}_{k} \otimes \mathbf{e}_{l} \\
& \mathcal{I}_{n+1}=\frac{1}{2}\left(\delta_{i k} \tau_{j l}+\tau_{i k} \delta_{j l}+\delta_{i l} \tau_{j k}+\tau_{i l} \delta_{j k}\right)_{n+1} \mathbf{e}_{i} \otimes \mathbf{e}_{j} \otimes \mathbf{e}_{k} \otimes \mathbf{e}_{l}
\end{aligned}
$$

and $\mathbf{e}_{k}(k=1,2,3)$ are the unit vectors of the Cartesian basis. The Kirchhoff stress in Eq. (6.4) is determined from the stress transformation law, i.e.

$$
\boldsymbol{\tau}_{n+1}=\mathbf{F}_{n+1} \mathbf{S}_{n+1} \mathbf{F}_{n+1}^{\mathrm{T}}
$$

where the second Piola-Kirchhoff stress, $\mathbf{S}_{n+1}$ is calculated utilizing Eq. (4.1).

Substitution of Eq. (6.2) into Eq. (6.7) $)_{1}$ results in the following form of the material stiffness in the current configuration

$$
\mathcal{C}_{n+1}^{\tau c}=\mathcal{C}_{n+1}^{v o l}+\left[1+\sum_{k=1}^{P} \gamma_{k}\left(1+\frac{\Delta z_{n+1}}{2 \widetilde{D}_{k}}\right)^{-1}+\sum_{j=1}^{N} \Gamma_{j} \frac{1-\mathrm{e}^{-\frac{\Delta t}{\tau_{j}}}}{\frac{\Delta t}{\tau_{j}}}\right] \mathcal{C}_{n+1}^{i s o}
$$

where

$$
\mathcal{C}^{\text {vol }}=\frac{2}{D_{1}} J(J-1)(\mathbf{1} \otimes \mathbf{1}-2 \mathbf{I})+J^{2} \frac{2}{D_{1}} \mathbf{1} \otimes \mathbf{1}
$$


and

$$
\begin{aligned}
\mathcal{C}^{i s o} & =\frac{2}{3} \mu\left[1+\frac{b}{\kappa}\left(\bar{I}_{1}-3\right)\right]^{\kappa-1} \bar{I}_{1}\left(\mathbf{I}+\frac{1}{3} \mathbf{1} \otimes \mathbf{1}\right)-\frac{2}{3} J^{-\frac{2}{3}} \mu\left[1+\frac{b}{\kappa}\left(\bar{I}_{1}-3\right)\right]^{\kappa-1}(\mathbf{B} \otimes \mathbf{1}+\mathbf{1} \otimes \mathbf{B}) \\
& +2 J^{-\frac{4}{3}} \mu \frac{b(\kappa-1)}{\kappa}\left[1+\frac{b}{\kappa}\left(\bar{I}_{1}-3\right)\right]^{\kappa-2} \mathbf{B} \otimes \mathbf{B} \\
& -\frac{2}{3} J^{-\frac{2}{3}} \mu \frac{b(\kappa-1)}{\kappa}\left[1+\frac{b}{\kappa}\left(\bar{I}_{1}-3\right)\right]^{\kappa-2} \bar{I}_{1}(\mathbf{B} \otimes \mathbf{1}+\mathbf{1} \otimes \mathbf{B}) \\
& +\frac{2}{9} \mu \frac{b(\kappa-1)}{\kappa}\left[1+\frac{b}{\kappa}\left(\bar{I}_{1}-3\right)\right]^{\kappa-2} \bar{I}_{1}^{2} \mathbf{1} \otimes \mathbf{1}
\end{aligned}
$$

are, respectively, the volumetric and isochoric components of the elasticity tensor corresponding to the stored-energy functions as given in Eqs (3.11), cf Suchocki (2011).

The viscoelastic-plastic model has been implemented into FE software Abaqus by taking advantage of the user subroutine UMAT (UserMATerial), which is called by the FE solver during every iteration of the Newton-Raphson numerical procedure (Hibbit et al. 2008). The written subroutine calculates Cauchy stress tensor and material Jacobian defined in Eq. (6.6) for each finite element. These quantities are subsequently used by Abaqus to form up the element stiffness matrix. Finally, the global stiffness matrix is assembled by Abaqus using the element stiffness matrices. The calculations done by the written subroutine UMAT are listed further in the text. The user subroutines used in other FE programs to define custom constitutive equations have a similar structure.

In order to verify the performance of the developed UMAT code, several exemplary simulations have been performed using the Abaqus FEM program. The simulations involved a $15 \mathrm{~mm} \times 15 \mathrm{~mm} \times 15 \mathrm{~mm}$ UHMWPE block undergoing ramp tension test, ramp compression test and sinusoidal deformation with variable amplitude. In the first approach, the polymeric block is meshed using a single finite element $\mathrm{C} 3 \mathrm{D} 8 \mathrm{H}^{1}$. Subsequently, all the simulations are conducted using the polyethylene block meshed with 125 elements. In each of the simulated processes the excitation was kinematic, i.e. the frontal face of the block performs a designated displacement program. The displacement distribution and the used boundary conditions are depicted in Fig. 4. Due to the assumed incompressibility, the deformation gradient tensor has the following components

$$
\mathbf{F}_{3 \times 3}=\left[\begin{array}{ccc}
\lambda_{1} & 0 & 0 \\
0 & \frac{1}{\sqrt{\lambda_{1}}} & 0 \\
0 & 0 & \frac{1}{\sqrt{\lambda_{1}}}
\end{array}\right]
$$

where $\lambda_{1}$ denotes the stretch ratio in the direction $x$ (Fig. 4).

(a)

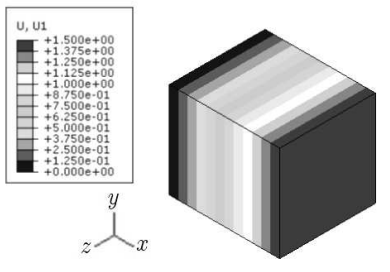

(b)

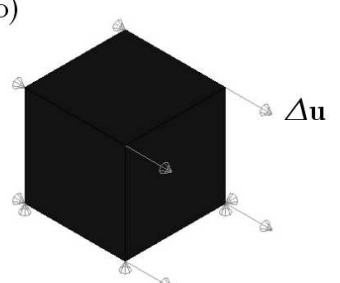

Fig. 4. Homogeneous deformation of a single finite element. (a) Distribution of the displacement component in the tension/compression direction. (b) Applied boundary conditions

All the simulations have been repeated utilizing programs written in MATLAB in which the discretized equations presented in Section 4 are used to calculate the stresses for the given deformation histories.

\footnotetext{
${ }^{1}$ Cubic, three-dimensional, 8 nodes, hybrid.
} 

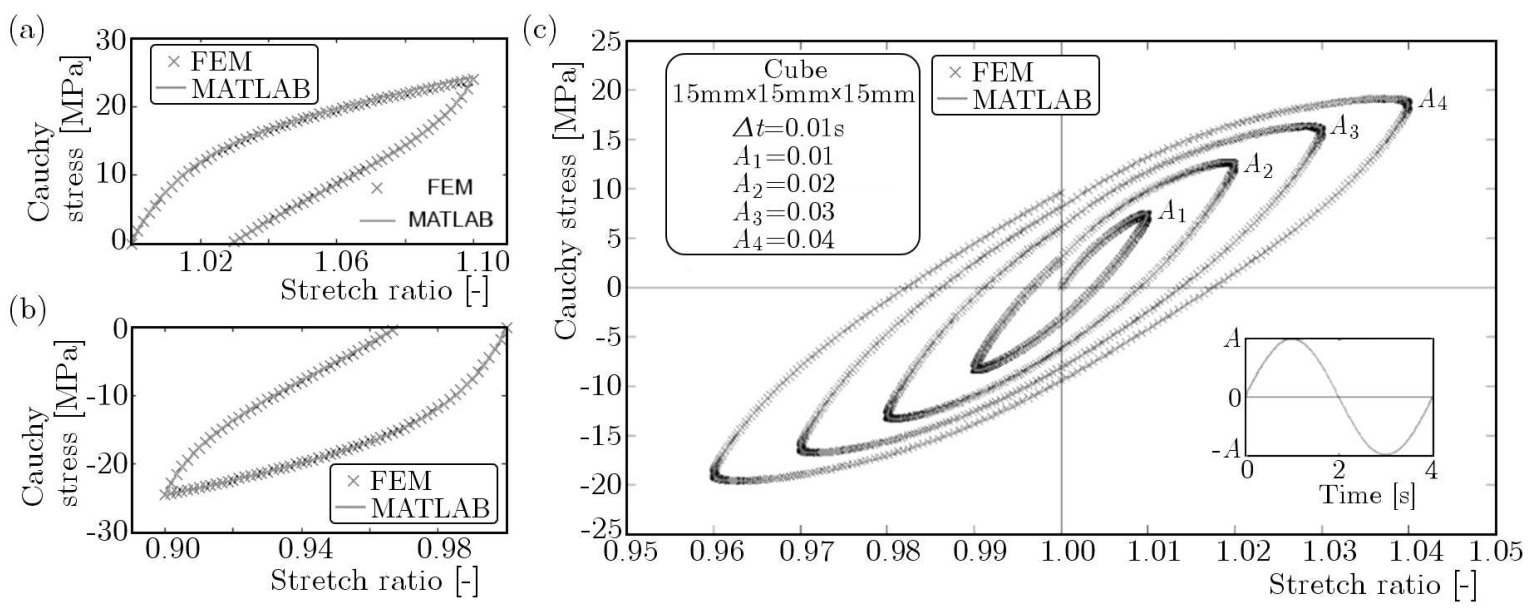

Fig. 5. Polyethylene block undergoing (a) ramp tension and (b) ramp compression tests with the deformation rate of $0.003 \mathrm{~s}^{-1}$ and (c) cyclic deformation history - a sinusoidal function with the increasing amplitude $A$

\section{Algorithm for the implementation in Abaqus}

Input: $\mathbf{F}_{n+1}$, no. of direct and shear stress components

1. Calculate strain measures from the current increment

$$
\mathbf{C}_{n+1}=\mathbf{F}_{n+1}^{\mathrm{T}} \mathbf{F}_{n+1} \quad \overline{\mathbf{F}}_{n+1}=J_{n+1}^{-\frac{1}{3}} \mathbf{F}_{n+1} \quad \overline{\mathbf{B}}_{n+1}=\overline{\mathbf{F}}_{n+1} \overline{\mathbf{F}}_{n+1}^{\mathrm{T}}
$$

2. Extract variables from the previous increment

3. Calculate elastic stresses from the current increment

$$
\begin{array}{ll}
\mathbf{S}_{0 n+1}^{v o l}=J_{n+1} p_{n+1} \mathbf{C}_{n+1}^{-1} & p_{n+1}=\partial_{J_{n+1}} U\left(J_{n+1}\right) \\
\mathbf{S}_{0 n+1}^{i s o}=J_{n+1}^{-\frac{2}{3}} \operatorname{DEV}\left[\overline{\mathbf{S}}_{n+1}\right] & \overline{\mathbf{S}}_{n+1}=2 \partial_{\overline{\mathbf{C}}_{n+1}} \bar{W}\left(\overline{\mathbf{C}}_{n+1}\right) \\
\mathbf{S}_{0 n+1}=\mathbf{S}_{0 n+1}^{v o l}+\mathbf{S}_{0 n+1}^{i s o} &
\end{array}
$$

4. Update endochronic and viscoelastic overstresses $(k=1,2, \ldots, P)$,

$$
\begin{aligned}
& (j=1,2, \ldots, N) \\
& \Delta z_{n+1}=\left(\Delta \overline{\mathbf{C}}_{n+1} \cdot \Delta \overline{\mathbf{C}}_{n+1}\right)^{\frac{1}{2}} \quad \Delta \overline{\mathbf{C}}_{n+1}=\overline{\mathbf{C}}_{n+1}-\overline{\mathbf{C}}_{n} \\
& \widetilde{\mathbf{H}}_{k n+1}=\frac{\left(1-\frac{1}{\widetilde{D}_{k}} \frac{\Delta z_{n+1}}{2}\right) \widetilde{\mathbf{H}}_{k n}+\gamma_{k}\left(\mathbf{S}_{0 n+1}^{i s o}-\mathbf{S}_{0 n}^{i s o}\right)}{1+\frac{1}{D_{k}} \frac{\Delta z_{n+1}}{2}} \\
& \mathbf{H}_{j n+1}=\mathrm{e}^{-\frac{\Delta t}{\tau_{j}}} \mathbf{H}_{j n}+\Gamma_{j} \frac{1-\mathrm{e}^{-\frac{\Delta t}{\tau_{j}}}}{\frac{\Delta t}{\tau_{j}}}\left(\mathbf{S}_{0 n+1}^{i s o}-\mathbf{S}_{0 n}^{i s o}\right)
\end{aligned}
$$

5. Calculate total stress from the current increment

$$
\mathbf{S}_{n+1}=\mathbf{S}_{0 n+1}+\sum_{k=1}^{P} \widetilde{\mathbf{H}}_{k n+1}+\sum_{j=1}^{N} \mathbf{H}_{j n+1} \quad \boldsymbol{\sigma}_{n+1}=\frac{1}{J_{n+1}} \mathbf{F}_{n+1} \mathbf{S}_{n+1} \mathbf{F}_{n+1}^{\mathrm{T}}
$$

6. Calculate viscoelastic-plastic stiffness from the current increment

7. Store stresses and isochoric C-G tensor from the current increment 
In Fig. 5, the results of ramp tension (Fig. 5a) and compression (Fig. 5b) are shown. In the case of ramp tension simulation, the material is deformed at a constant rate $\dot{\lambda}_{1}=-0.003 \mathrm{~s}^{-1}$ until the stretch ratio $\lambda_{1}=1.1$ is reached. Subsequently, unloading is performed at the deformation rate of $\dot{\lambda}_{1}=0.003 \mathrm{~s}^{-1}$. The compression process was simulated analogously.

In Fig. 5c, the results of sinusoidal loading simulation are presented. The simulated process comprises of four periods, each performed at different amplitude.

The predictions of the FEM simulations utilizing UMAT are in a good agreement with the results produced by MATLAB programs (see Fig. 5). All the simulations have been performed utilizing the material parameter values collected in Table 1.

\section{Conclusions}

In this study, a new viscoelastic-plastic model for thermoplastic polymers and resins is presented. The model is formulated by utilizing the formalism of stress-like internal state variables. It has been implemented into the FE software Abaqus and applied to model the inelastic behavior of polyethylene with very good results.

\section{References}

1. Abdul-Hameed H., Messager T., Zaïri F., Nä̈т-Abdelaziz M., 2014, Large-strain viscoelastic-viscoplastic constitutive modeling of semi-crystalline polymers and model identification by deterministic/evolutionary approach, Computational Materials Science, 90, 241-252

2. Alkas Yonan S., Soyarslan C., Haupt P., Kwiatkowski L., Tekkaya A.E., 2013, A simple finite strain non-linear visco-plastic model for thermoplastics and its application to the simulation of incremental cold forming of polyvinylchloride (PVC), International Journal of Mechanical Sciences, 66, 192-201

3. Ayoub G., Zaïri F., Fréderix C., Gloaguen J.M., Naїт-Abdelaziz M., Seguela R., LefeBvre J.M., 2011, Effects of crystal content on the mechanical behaviour of polyethylene under finite strains: experiments and constitutive modelling, International Journal of Plasticity, $\mathbf{2 7}, 492-511$

4. BARDELla L., 2001, A phenomenological constitutive law for the nonlinear viscoelastic behaviour of epoxy resins in the glassy state, European Journal of Mechanics - A/Solids, 20, 907-924

5. Ben Hadj Hamouda H., Laiarinandrasana L., Piques R., 2007, Viscoplastic behaviour of a medium density polyethylene (MDPE): constitutive equations based on double nonlinear deformation model, International Journal of Plasticity, 23, 1307-1327

6. Bergström J.S., Bischoff J.E., 2010, An advanced thermomechanical constitutive model for UHMWPE, International Journal of Structural Changes in Solids, 2, 1, 31-39

7. Drozdov A.D., Christiansen J. De C., 2007, Cyclic viscoplasticity of high-density polyethylene/montmorillonite clay nanocomposite, European Polymer Journal, 43, 1, 10-25

8. Hassan T., Colak O.U., Clayton P.M., 2011, Uniaxial strain and stress-controlled cyclic responses of ultrahigh molecular weigth polyethylene: experiments and model simulations, Journal of Engineering Materials and Technology, 133, 021010-1-021010-9

9. Hibbit B., Karlsson B., Sorensen P., 2008, ABAQUS Theory Manual, Hibbit, Karlsson \& Sorensen Inc.

10. Holzapfel G.A., 2010, Nonlinear Solid Mechanics, John Wiley \& Sons Ltd., New York

11. Knowles J.K., 1977, The finite anti-plane shear field near the tip of a crack for a class of incompressible elastic solids, International Journal of Fracture, 13, 5, 611-639 
12. Krairi A., Doghri I., 2014, A thermodynamically-based constitutive model for thermoplastic polymers coupling viscoelasticity, viscoplasticity and ductile damage, International Journal of Plasticity, 60, 163-181

13. Kästner M., Obst M., Brummund J., Thielsch K., Ulbricht V., 2012, Inelastic behavior of polymers - experimental characterization, formulation and implementation of a material model, Mechanics of Materials, 52, 40-57

14. Pipkin A.C., Rivlin R.S., 1965, Mechanics of rate-independent materials, ZAMP, 16, 3, 313-327

15. Suchocki C., 2011, A finite element implementation of Knowles stored-energy function: theory, coding and applications, The Archive of Mechanical Engineering, 58, 319-346

16. Suchocki C., 2013, A quasi-linear viscoelastic rheological model for thermoplastics and resins, Journal of Theoretical and Applied Mechanics, 51, 1, 117-129

17. Taylor R.L., Pister K.S., Goudreau G.L., 1970, Thermomechanical analysis of viscoelastic solids, International Journal for Numerical Methods in Engineering, 2, 45-59

18. Wu H.C., 2005, Continuum Mechanics and Plasticity, CRC Press, New York 